\title{
Detection of melanocyte lineage-specific genes in vitiligo lesions
}

\author{
WANWEN XU ${ }^{1}$ and XIONG WANG ${ }^{2}$ \\ ${ }^{1}$ Division of Dermatology, Wuhan Third Hospital and Tongren Hospital of Wuhan University; \\ ${ }^{2}$ Division of Nephrology, Renmin Hospital, Wuhan University, Wuhan, Hubei 430060, P.R. China
}

Received January 29, 2018; Accepted October 19, 2018

DOI: $10.3892 /$ etm.2019.7496

\begin{abstract}
Vitiligo is an acquired pigmentary disorder characterized by the loss of skin color and functional melanocytes. The pathogenesis of vitiligo remains unclear, which means that effective clinical treatment is difficult. However, if melanocyte linkage-specific genes are identified in vitiligo lesions, the appropriate treament for melanocytes may be implemented. The current study aimed to detect the expression of melanocyte lineage-specific genes in vitiligo lesion needle biopsies and to predict the occurrence of perifollicular repigmentation in depigmented macules. A total of 6 patients with stable vitiligo and 4 healthy volunteers were recruited from the Department of Dermatology, Wuhan Third Hospital. Total RNA was extracted from skin tissue needle biopsies from the periphery and center of depigmented macules, and adjacent normal skin. The expression of dopachrome tautomerase (Dct), tyrosinase (Tyr) and $\beta$-actin (ACTB) genes were detected using a reverse transcription (RT)-semi quantitative polymerase chain reaction (PCR) assay. RNA extracted from $7 \mathrm{mg}$ of skin samples was sufficient to amplify all three genes. The expression profile of each patient was then observed in the center of vitiligous lesions and were deemed to be Dct+Tyr-ACTB+, Dct-Tyr-ACTB+ or Dct+Tyr+ACTB+. At 5 months, patient follow-up indicated that perifollicular repigmentation was efficaciously induced by $308 \mathrm{~nm}$ eximer light radiation in a patient who had a Dct+Tyr-ACTB+ gene expression. The results demonstrated that needle skin biopsy and RT-semi quantitative PCR may be used as a less traumatic and reliable method to detect the expression profiles of melanocyte lineage-specific genes in needle biopsies, which may have the potential to predict the occurrence of perifollicular repigmentation in vitiligo.
\end{abstract}

Correspondence to: Dr Wanwen Xu, Division of Dermatology, Wuhan Third Hospital and Tongren Hospital of Wuhan University, 241 Pengliuyang Road, Wuhan, Hubei 430060, P.R. China

E-mail: xuww9905@126.com

Key words: vitiligo, melanocyte, tyrosinase, dopachrome tautomerase, perifollicular repigmentation

\section{Introduction}

Vitiligo is a common acquired skin disease characterised by a loss of skin color that may occur at any age but most commonly occurs in young adults (1). Vitiligo can arise in any area of the skin, but mostly presents in exposed regions and those where rubbing occurs (2). This disease does not cause significant physical disorder of the affected skin; however, severe effects on the physical and mental health of patients may occur, hindering their ability to work and communicate socially (3). The pathogenesis of vitiligo remains unclear, which makes its clinical treatment more challenging. Since hair follicle cells are considered to be immune privileged (4), melanocyte precusors that are stored in this area may be protected from disease. It is therefore critical to induce perifollicular repigmentation in vitiligo lesions of hair-covered skin. It has been demonstrated that the rate of ineffectiveness in patients with vitiligo may be as high as $50 \%(5,6)$, which may be associated with the possibility that clinicians cannot assess the presence of potentially mature melanocytes in vitiligo hair follicles prior to treatment.

If melanocyte linkage-specific genes are identified in vitiligo lesions, the appropriate treament measure may be implemented for melanocytes. Tyrosinase (Tyr), tyrosine related protein (TRP)-1 and TRP-2 are important enzymes for melanin synthesis, with Tyr being the most vital (7) Tyr possesses tyrosine hydroxylase and dopa oxidase activities and functions to promote the synthesis of dopaquinone from L-tyrosine (8). Tyr activity is positively associated with melanin synthesis in melanocytes and the regulation of Tyr activity mediates melanin production (9). In addition, Tyr activity in vitiligo lesions has been determined to be lower than that of normal skin (8). Furthermore, Tyr is only expressed in differentiated mature melanocytes (9). TRP-2, also known as Dopachrome tautomerase (Dct), is a recognized biomarker for melanocyte stem cells (10). The current study aimed to assess the expression of melanocyte-specific biomarker genes (Tyr and Dct) and proteins in needle biopsies of vitiligo lesions using reverse transcription (RT)-semi-quantitative polymerase chain reaction (PCR), the results of which may help establish an accurate and minimally invasive method for the prediction of perifollicular repigmentation in vitiligo lesions.

\section{Materials and methods}

Clinical data. The present study was approved by the Medical Ethics Committee of Wuhan ThirdHospitaland Tongren Hospital 
of Wuhan University (Wuhan, China). A total of 4 healthy volunteers ( 2 males and 2 females; age range, 24-50 years old) and 6 patients with static stage vitiligo ( 3 males and 3 females; age range, 21-65 years old) diagnosed by the Department of Dermatology at Wuhan Third Hospital were enrolled from January 2012 to December 2017. Written informed consent was obtained from all participants. The inclusion criteria were as follows: Patients with vitiligo in stationary phases, aged 18-70. The exclusion criteria were as follows: Patients with a family history of vitiligo or autoimmune diseases, or patients who had undergone early ultraviolet treatment.

Needle biopsy. The skin of the affected area was disinfected with conventional active iodine solution and deiodinated using $75 \%$ ethanol. The vitiligo lesion and surrounding normal skin were exposed under surgical towel and biopsies were collected from the center of the whitened area, the edge of the whitened area and the surrounding normal skin. A total of three skin tissue specimens were taken per patient; 18 skin specimens were obtained from six patients with vitiligo. A total of $0.5 \mathrm{ml}$ $2 \%$ lidocaine solution was drawn into a 1-ml syringe and subcutenanously injected to form a skin pimple, which was then gently lifted using a needle tip. The epidermis $(\sim 3$ or $7 \mathrm{mg}$ samples) of the pimple was cut with a scalpel parallel to the surface of the skin (Fig. 1A-D). If point bleeding occurred, the area was pressed with a sterilized gauze. Skin tissue specimens were then placed directly into an eppendorf (EP) tube with $50 \mu 1$ of cold TRIzol reagent (Invitogen; Thermo Fisher Scientific, Inc., Waltham, MA, USA) and weighed using an analytical balance and then homogenized by micro tissue grinders (Kimble Chase Life Science and Research Products LLC, Rockwood, TN, USA; Fig. 1E). To avoid genetic contamination, all tools (syringe, scalpel and gauze) were changed for each specimen.

RT-semi-quantitative PCR. Skin specimens were homogenized in the EP tube using a Pellet Pestle Cordless Motor (Kimble Chase Life Science and Research Products LLC). Total RNA was extracted using Trizol reagent following the manufacturer's protocol. To increase the efficiency of RNA extraction, all reagents and equipment were RNAse free the RNA concentration was measured at $260 \mathrm{~nm}$ to calculate the A260/A280. First strand cDNA was synthesized using a M-MLV Reverse Transcription kit at $42^{\circ} \mathrm{C}$ for $40 \mathrm{~min}$ (Invitrogen; Thermo Fisher Scientific, Inc.). PCR was performed using DreamTaq DNA polymerase (cat. no. EP0702; Thermo Fisher Scientific, Inc.) with the following thermocycling conditions: Initial denaturation at $94^{\circ} \mathrm{C}$ for $2 \mathrm{~min}$; denaturation for 35 cycles of $94^{\circ} \mathrm{C}$ for $30 \mathrm{sec}$; annealing at $55^{\circ} \mathrm{C}$ for $30 \mathrm{sec}$ and extension at $72^{\circ} \mathrm{C}$ for $30 \mathrm{sec}$; and extension at $72^{\circ} \mathrm{C}$ for $2 \mathrm{~min}$. The product was then separated on a $2 \%$ agarose gel. The bands were visualized using ethidium bromide. The specific primers utilized for PCR were as follows: $\beta$-actin (internal control), forward 5'-AGCGAGCATCCCCCAAAGTT-3' and reverse 5'-GGGCACGAAGGCTCATCATT-3', with a PCR product of 285 bp; Dct, forward 5'-AGTGATTCGGCAGAACATCC-3' and reverse 5'-AGTTCCAGTAGGGCAAAGCA-3', with a PCR product of $368 \mathrm{bp}$; Tyr, forward 5'-CAGCTTTCAGGC AGAGGTTC-3' and reverse 5'-GCTTCATGGGCAAAATCA AT-3', with a PCR product of $470 \mathrm{bp}$.
Immunostaining. Tyr and Dct protein expression from tissue obtained from the biopsy was assessed using immunohistochemistry. The tissues were fixed with $4 \%$ formalin for $24 \mathrm{~h}$ at room temperature, dehydrated in different concentrations (50, 70, 80, 95 and 100\%) of ethanol, paraffin-embedded and cut into $5-\mu$ m-thick sections. To deparaffinized the tissue sections, they were immersed in xylene two times ( $5 \mathrm{~min}$ each), then they were immersed in 100, 95, 75 and 50\% ethanol in turn ( 3 min each), washed in distilled water for $10 \mathrm{~min}$, washed in PBS two times (5 min each), and treated with $3 \% \mathrm{H}_{2} \mathrm{O}_{2}$ for $30 \mathrm{~min}$ at room temperature. Antigen retrieval for Tyr and Dct was performed by boiling the sections in citrate buffer $(0.01 \mathrm{~mol} / \mathrm{l}$; $\mathrm{pH} 6.0)$ for $10 \mathrm{~min}$ at $118^{\circ} \mathrm{C}$. Sections were incubated with $5 \%$ bovine serum albumin for $30 \mathrm{~min}$ at room temperature, and then incubated with rabbit anti-Tyr (cat. no. 251315; 1:100; Abbiotec, Inc., San Diego, CA, USA) and anti-Dct (cat. no. ab74073; 1:100; Abcam, Cambridge, UK) antibodies overnight at $4^{\circ} \mathrm{C}$. Sections were then washed with PBS and incubated with horseradish peroxidase-conjugated goat anti-rabbit immunoglobulin (Ig)G (cat. no. ab6721; 1:100; Abcam) for $1 \mathrm{~h}$ at room temperature. Following washing, peroxidase activity was visualized using DAB staining (Dako; Agilent Technologies, Inc., Santa Clara, CA, USA) for $1 \mathrm{~min}$ at room temperature and sections were counter-stained with haematoxylin for $30 \mathrm{sec}$ at room temperature. Images were captured using a light microscope at a magnification of x200. Negative controls were constructed by omitting the primary antibody or replacing it with normal rabbit IgG (cat. no. 2729; 1:100; Cell Signaling Technology, Inc., Danvers, MA, USA). Positive controls were constructed using the skin tissue from the healthy volunteers.

Light treatment and skin autografts. Vitiligo lesions were treated with $308 \mathrm{~nm}$ eximer light radiation eight times with TheraBeam UV308 equipment (USHIO, Inc., Tokyo, Japan) at $200 \mathrm{~mJ} / \mathrm{cm}^{2}$, each lasting 2-3 min. Skin autografts were peformed by an orthopedist, skin samples were taken from the inner thigh and transplanted to the forehead. Perifollicular repigmentation was evaluated by measurement the vilitigo lesion area.

Follow-up period. A total of 10 participants were followed up for 3 months. The participants came back to Wuhan Third Hospital once a week in the first month, but only the patients came back every 2 weeks during the second month. The follow up period was once per month after 2 months. One patient with vilitigo (male, 27 years old) was followed up for 5 months. The perifollicular repigmentation was observed by measuring the diameter and the area of vitiligo skin lesions.

\section{Results}

Needle biopsy method. Fig. 1A shows that $0.5 \mathrm{ml} 2 \%$ lidocaine was injected into the skin. The skin was then cut (Fig. 1B) and removed (Fig. 1C), and skin tissue ( 3 and $7 \mathrm{mg}$ ) retrieved via needle biopsy (Fig. 1D). The results demonstrated that Dct, Tyr and $\beta$-actin mRNA was detected in $7 \mathrm{mg}$ needle biopsy specimens, but not in the $3 \mathrm{mg}$ or epidermis specimens obtained via needle biopsy respectively (Fig. 1E). 


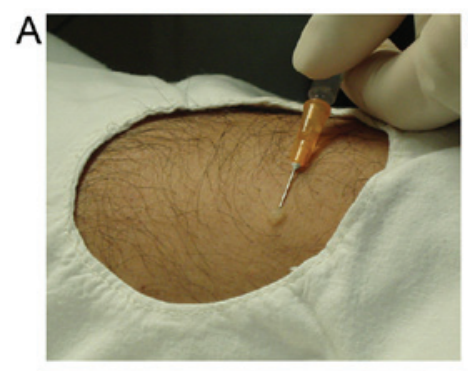

$\mathrm{D}$

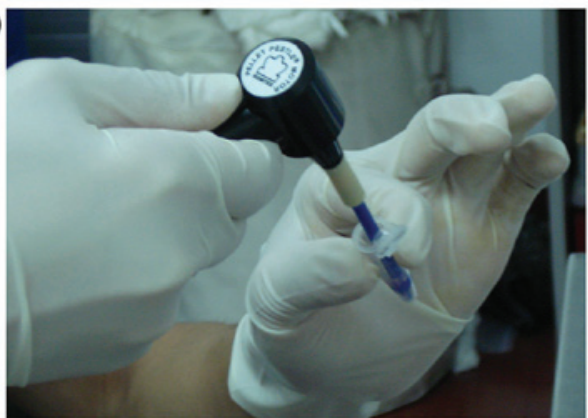

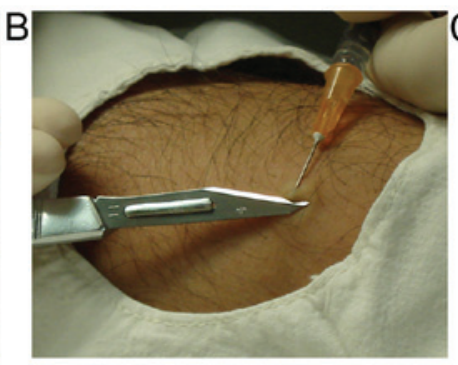

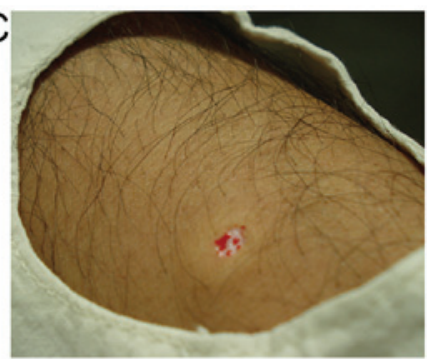

$\mathrm{E}$

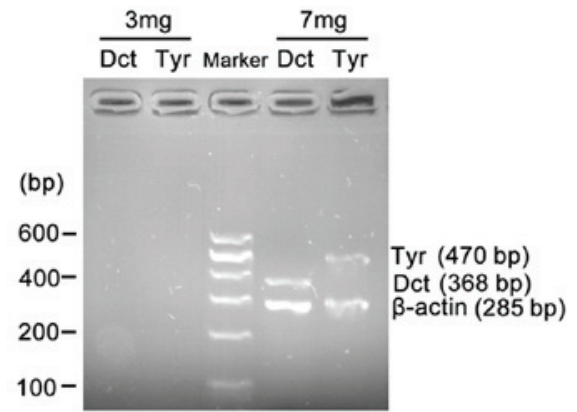

Figure 1. Sampling method. Needle biopsy were performed to obtain skin samples. (A) Lidocaine was injected subcutaneously in the target area. (B) A skin sample was obtained. (C) The wound postoperation. (D) A pellet pestle cordless motor was used to RNA extraction. (E) Dct, Tyr and $\beta$-actin mRNA expression in tissue samples; a DNA marker. Dct, dopachrome tautomerase; Tyr, tyrosinase.
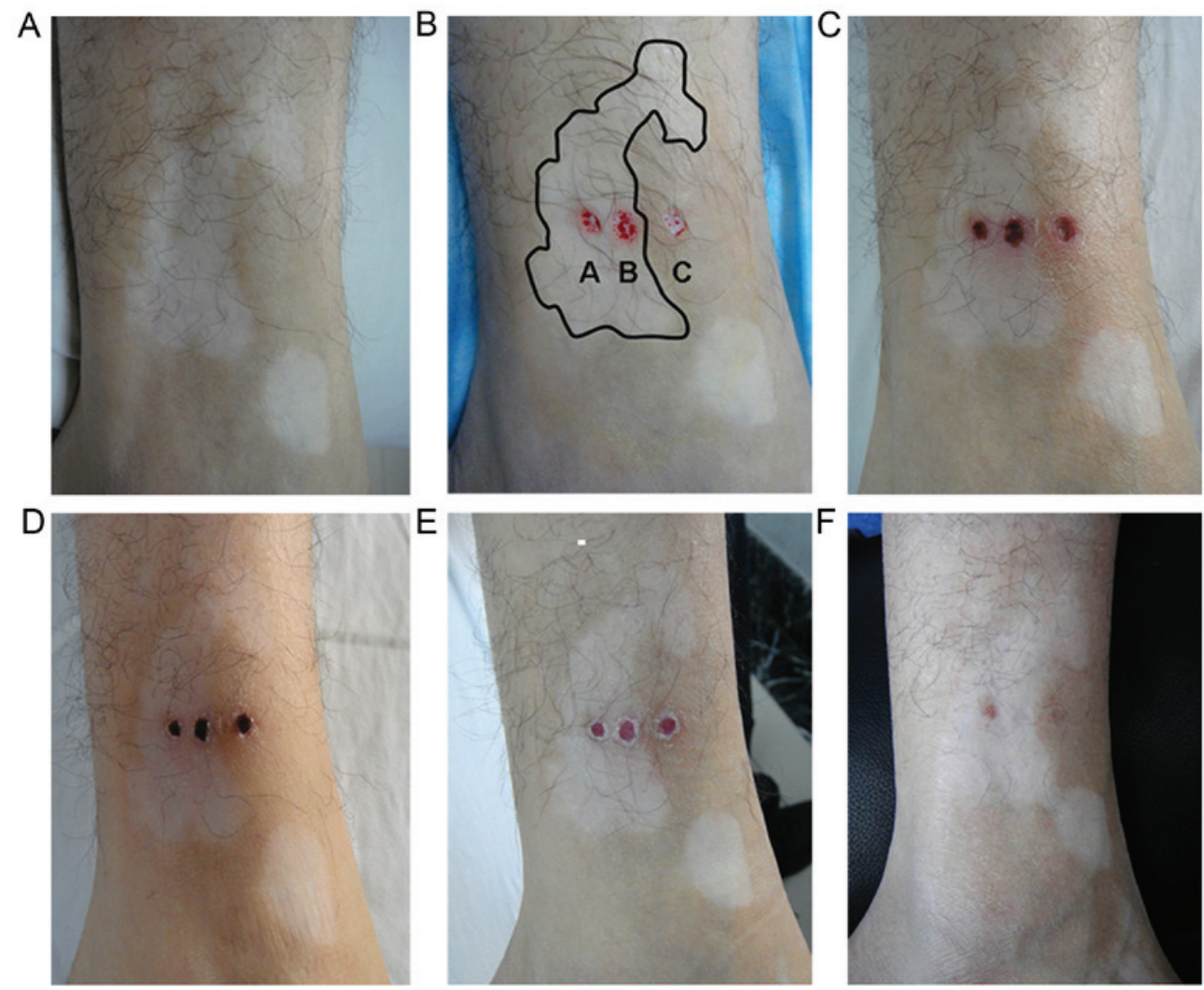

Figure 2. Needle biopsy wound healing. (A) Representative images obtained from a patient with vitiligo (male, 27 years old, white patches above left ankle joint for 3 years) who received needle biopsy. (B) Position of the needle biopsy. The black border represents the area of vitiligo. A, centre of the lesion; B, edge of lesion; and C, surrounding normal skin. Wound healing following sampling at (C) 5 days, (D) 2 weeks, (E) 3 weeks and (F) 1 month.

Needle biopsy wound healing. The healing of the wounds created following needle biopsy in the 10 participants was followed up for 3-5 months. Following one month, the wound was nearly healed without the formation of scar (Fig. 2).
Detection of melanocyte lineage-specific genes in vitiligo lesion needle biopsies. The expression of Dct, Tyr and $\beta$-actin was detected in 18 skin specimens obtained from six patients with vitiligo (three specimens per patient). As needle biopsies 
A

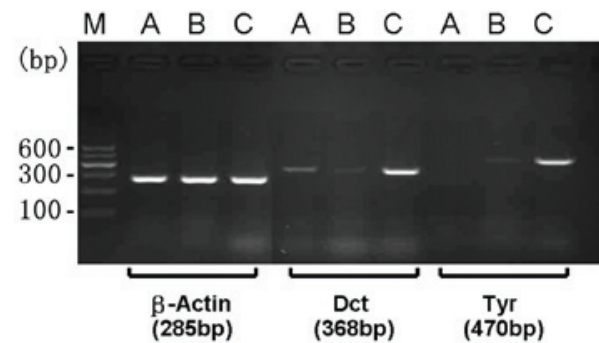

C

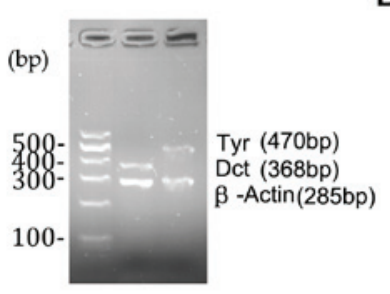

$\mathrm{F}$

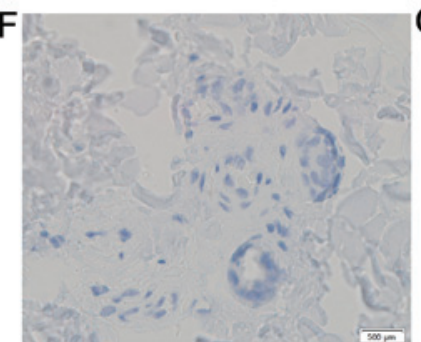

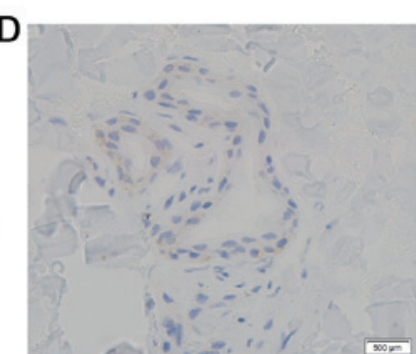

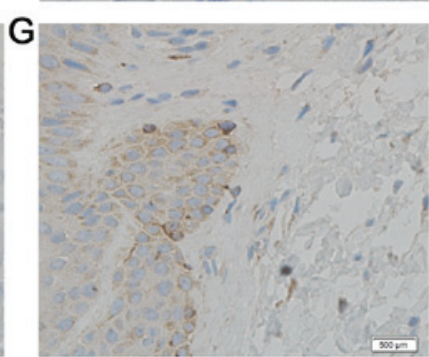

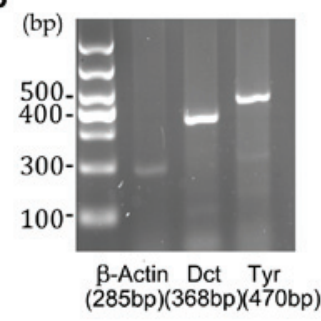
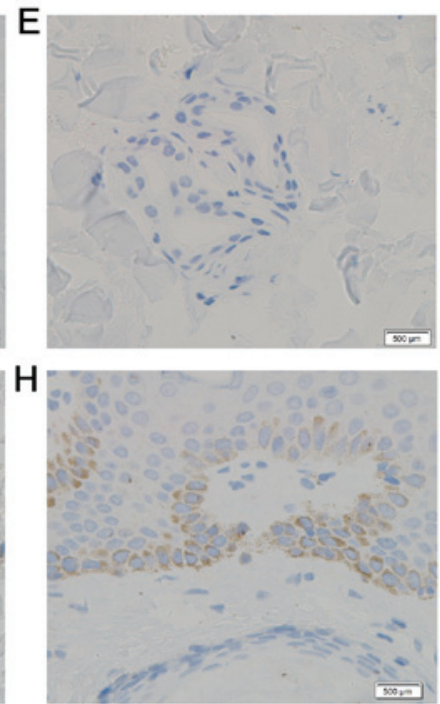

Figure 3. Expression of Dct, Tyr and $\beta$-actin in a patient with vitiligo as determined by reverse transcription-semi quantitative PCR. (A) PCR result (Dct+Tyr-ACTB+) of one patient (male, 27 years old) with vitiligo. Lanes were labelled accordingly: A, the centre of lesion; B, the edge of lesion; C, surrounding normal skin. (B) PCR results obtained from one healthy volunteer (male, 25 years old). (C) PCR results (Dct+Tyr+ACTB+) of a patient (female, 32 years old) with vilitigo. Immunostaning results were (D) Dct+ (male, 27 years old), (E) Tyr- (male, 27 years old), (F) negative control (male, 27 years old). (G) Dct+ (healthy male, 36 years old) and $(\mathrm{H}) \mathrm{Tyr}+$ (healthy male, 36 years old) were positive. The blue staining represents nuclei and brown staining represents positive protein staining. Scale bar=500 $\mu \mathrm{m}$. Dct, dopachrome tautomerase; Tyr, tyrosinase; PCR, polymerase chain reaction; ACTB, $\beta$-actin; M, DNA markers.

only retrieve a small quantity of skin tissue, the specimens were homogenized using a pellet pestle cordless motor, which markedly increased the efficiency of RNA extraction (data not shown). However, 1-10 $\mu \mathrm{g}$ of RNA were retrieved from $1 \mathrm{mg}$ of skin sample using TRIzol. Furthermore, the proportion of melanocytes and melanocyte stem cells was small in skin tissue, $\sim 10 \%$ of melanocytes appearing in the basal layer (data not shown).

In the centre of the vitiligo lesion, three patterns of Dct, Tyr and $\beta$-actin expression were detected: One case of Dct+Tyr$\beta$-actin (ACTB)+ (Fig. 3A); four cases of Dct-Tyr-ACTB+; and one case of Dct+Tyr+ACTB+ (Fig. 3C). Healthy volunteers presented with Dct+Tyr+ACTB+ (Fig. 3B). Measuring Dct and Tyr protein expression in the centre of the vitiligo lesion revealed Dct+ staining (Fig. 3D), Tyr staining was negative (Fig. 3E), no staining as the section was treated with PBS (Fig. 3F), the Dct positive control (Fig. 3G) and the Tyr positive control (Fig. 3H).

Light treatment and skin autografts. The results of light treatment in one patient with Dct+Tyr-ACTB+ was followed-up for 5 months. Following two treatments with $200 \mathrm{~mJ} / \mathrm{cm}^{2}$ using TheraBeam UV308 equipment after the needle biopsy, perifollicular repigmentation was observed within the vitiligo lesion area after 3 months (Fig. 4A and B). In a second patient with Dct+Tyr+ACTB+, who was treated the same as aforementioned, but with eight treatments, perifollicular repigmentation was observed within the vitiligo lesion area following treatment (Fig. 4C and D). In 3 patients with Dct-Tyr-ACTB+, perifollicular repigmentation was also observed following skin treatment autograft (Fig. 4E and F; representative image of 1 patient). In 1 patient with Dct-Tyr-ACTB+, no perifollicular repigmentation was detected following treatment with $200 \mathrm{~mJ} / \mathrm{cm}^{2}$ eight times (Fig. 4G and H).

\section{Discussion}

The pathogenesis of vitiligo is complex and its clinical treatments are diverse, with patients recieving local topical or oral corticosteroids, immunomodulators, phototherapy and surgery (11). The re-coloring mechanism of vitiligo has not yet been fully elucidated, but a previous study have demonstrated that the most important mechanism includes re-coloring that expands outward from the center of the hair follicle (12). For the effective non-dermatoplastic treatment of vitiligo, most theapies require live melanocytes around hair follicles, as live melanocytes are the source of the melanin (13). Melanocyte stem cells are stored in the hair follicle and funtion to proliferate, migrate towards the epidermis and synthesize melanin following stimulation (13). Melanin is then transported to keratinocytes to induce vitiligo lesion re-coloring (13). Therefore, it is critical to detect the presence of melanocyte 

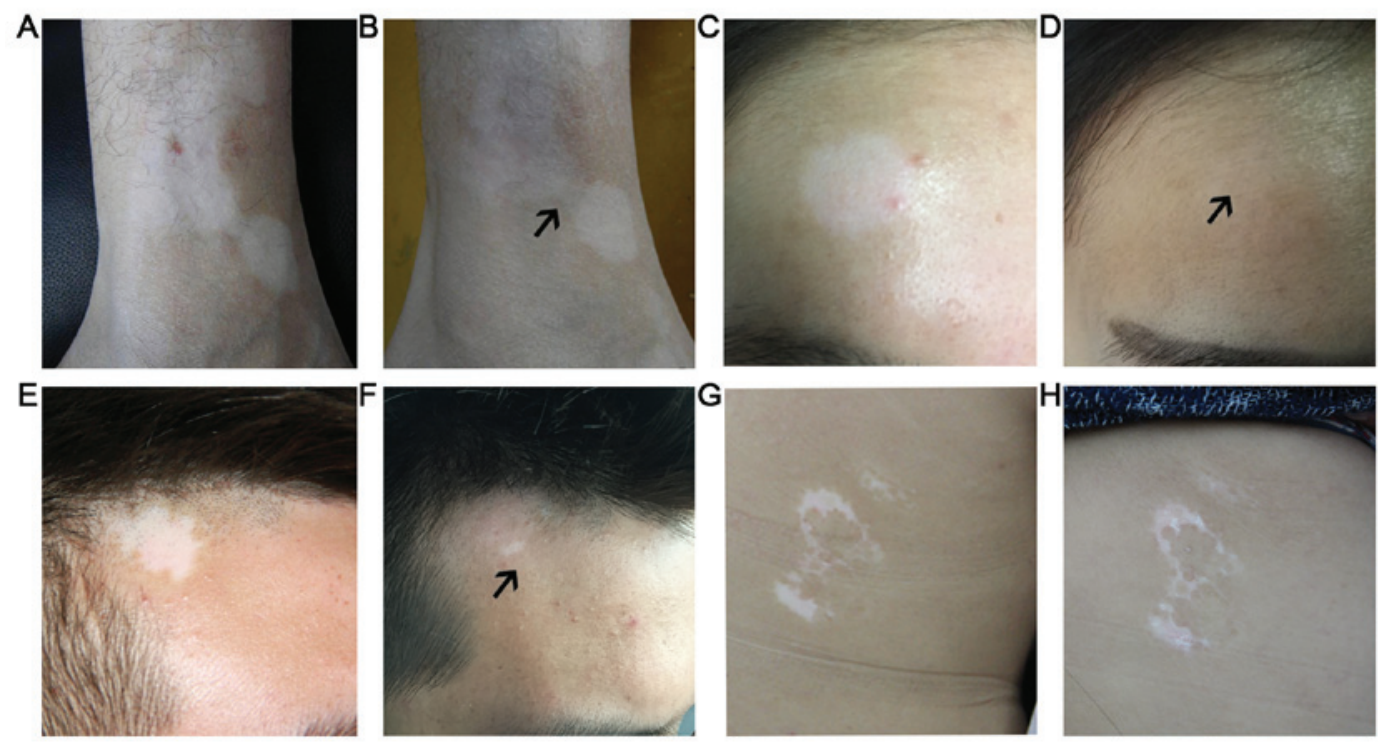

Figure 4. Several patients received different treatments to induce perifollicular repigmentation. The patient (male, 27 years old) was treated twice using TheraBeam UV308 equipment at $200 \mathrm{~mJ} / \mathrm{cm}^{2}$ (2-3 mins each. Lesion (A) prior to and (B) following light treatment. A second patient (female, 32 years old) with a Dct+Tyr+ACTB+ expression profile was assessed. Following eight treatments at $200 \mathrm{~mJ} / \mathrm{cm}^{2}$ using TheraBeam UV308 equipment, Hair follicle re-coloring was observed in the vitiligo lesion area. Lesion (C) prior to and (D) following light treatment. In a patient (male, 35 years old) exhibiting Dct-Tyr-ACTB+, perifollicular repigmentation was observed in the vitiligo lesion following a skin autograft. Lesion (E) prior to and (F) following autograft. In one patient (female, 42 years old) with the Dct-Tyr-ACTB+ expression profile, no perifollicular repigmentation was observed following eight treatments at $200 \mathrm{~mJ} / \mathrm{cm}^{2}$ using TheraBeam UV308. Lesions $(\mathrm{G})$ prior to and $(\mathrm{H})$ following light treatment. Black arrows indicate perifollicular repigmentation. Dct, dopachrome tautomerase; Tyr, tyrosinase; ACTB, $\beta$-actin.

stem cells around the hair follicle, which will help determine the possibility of hair follicle re-coloring in vitiligo lesions. However, a non-invasive method for the clinical detection of melanocyte presence has yet to be elucidated. The current study therefore aimed to establish an effective method for the detection of melanocyte linkage-specific gene expression in order to determine the presence of melanocyte stem cells.

Previous studies revealed that Dct mutations severely affect the maturation and development of late melanin bodies $(14,15)$. Therefore, the present study also aimed to assess the presence of melanocyte stem cells or melanocytes through the expression of Tyr and Dct in vitiligo lesions.

To detect melanocyte gene expression in vitiligo lesions, it is necessary to establish a minimally invasive and effective method for the collection of skin specimens. Suction blistering is a common method for skin sampling in dermatology and is often used in autologous skin tranplantations to treat patients with vitiligo $(5,16,17)$. To collect dermal skin tissue, the current study utilized a needle biopsy to ensure that sampling occurred where melanocyte stem cells were located (the hair follicle area). This was then used to detect the expression of the melanocyte-specific gene, Dct, in retrieved tissues. The results demonstrated that the expression of melanocyte-specific genes was detected in $7 \mathrm{mg}$ of normal skin tissues. Furthermore, the dynamic changes of wound healing were followed up following needle biopsy. Wound suppuration and scar formation were not exhibited, indicating that this method was safe and effective.

Since needle biopsy only retrieves a small quantity of skin tissue, a conventional homogenizer cannot homogenize the tissue completely and small quantities of homogenization solution may stick to the wall of tube, resulting in false-negative results. Therefore, all specimens retrieved by needle biopsy in the present study were homogenized using a pellet pestle cordless motor. To increase the sensitivity of screening for melanocyte-specific genes, it is critical to ensure the quality of sampling. In the present study, the differences in gene expression between 3 and $7 \mathrm{mg}$ normal skin samples from healthy volunteers were assessed. The results revealed that the expression of melanocyte-specific genes was not detected in $3 \mathrm{mg}$ specimens, but was observed in $7 \mathrm{mg}$ skin tissues, the latter of which meets experimental requirements. Therefore, $7 \mathrm{mg}$ skin specimens were determined to be the optimal size of skin tissue samples.

The current study further assessed the expression of Dct and Tyr in vitiligo lesion specimens. A previous study on hair growth in postnatal mice have determined that there are three types of melanocytes: i) Melanocytes that are located in the hair follicle area, only express Dct and are usually considered to be stem cells with the potential to proliferate and differentiate; ii) cell populations that are located outside the root sheath, which express Dct and Tyr, possess weak TRP-1 expression, exhibit proliferative activity and are deemed differentiated melanocytes; and iii) cell populations located in the hair matrix, close to the layer of dermal papilla, which express Dct, TRP-1 and Tyr genes, exhibit no proliferative activity but are able to melanin (18). According to the localization of melanin genes, Benzekri et al (19) proposed phase criteria for the assessment of vitiligo lesions, comprising three phases: Phase I, a partial loss of epidermal melanocytes with a possibility of re-coloring; phase II, complete loss of epidermal melanocytes, with the possibility of re-colouring via melanocytes stored in the hair follicle; and phase III, complete loss of melanocytes 
in the hair follicle with the impossibility of vitiligo lesion re-coloration without melanocyte transplantation. Based on the above criteria and the data of the current study, three possible results for the detection of melanocyte-specific genes in vitiligo lesions were determined: i) Dct+Tyr-. Dct is a recognized biomarker for melanocyte stem cells (10), but there is no Tyr gene expression in melanocyte stem cells. Dct and Tyr are both expressed in mature melanocyte (18). The Tyr- results indicated that there were no mature melanocytes in vitiligo lesions. The Dct+Tyr- results indicated a high possibility of melanocyte stem cell presence in vitiligo lesions and that treatment may activate the proliferation and differentiation of melanocyte stem cells, thus leading to hair follicle re-coloring. ii) Dct+Tyr+. Epidermal melanocyte cells and bulb portion melanocyte cells express both Tyr and Dct (18). This indicates the presence of melanocytes in vitiligo lesions; however, the loss of skin color means melanocytes may be dysfunctional. iii) Dct-Tyr- or Dct-Tyrt. These indicate the destruction of melanocytes and their stem cells or that there were no melanocytes in the vitiligo lesion, and a low possibility of hair follicle re-coloring $(10,20)$. In one case of Dct+Tyr-, hair follicle re-coloring occurred followng treatment with TheraBeam UV308 equipment. These results further support that the re-coloring of hair follicles requires the presence of melanocyte stem cells.

This current study established a method for the detection of vitiligo lesion-associated gene expression: Skin tissue samples were obtained by needle biopsies and melanocyte lineage-specific genes in vitiligo were detected. However, the number of patients was limited and an increased sample size should be utilized to verify the reliability of this method. Furthermore, future studies that assess hair follicle re-coloring in patients with vitiligo and the expression of melanocyte stem cell-specific genes are necessary to predict the significance of the results of the present study. The Dct gene is a biomarker of melanocyte stem cells and is highly expressed in adult melanocyte stem cells and mature melanocytes (10). Therefore, the detection of Dct gene expression will be helpful to assess the possibility of hair follicle re-coloring in vitiligo lesions. The proliferation and differentiation of melanocyte stem cells is regulated by Dct and other genes, including mdicrophthalmia-associated transcription factor (MITF) and paired box 3 (PAX3) (21). MITF is one of the earliest biomarkers for the development of melanoid differentiation into melanocytes (22). It regulates the expression of tyrosine family genes and participates in the regulation of melanogenesis, determining whether melanocytes are successfully differentiated or malignantly transformed to melanoma $(22,23)$. MITF-M, a 'melanocyte-specific' isoform, is selectively expressed in melanocyte lines (24), whereas the PAX3 gene is associated with undifferentiated melanocytes (21). As the current study isolated RNA using TRIzol reagent from $7 \mathrm{mg}$ skin specimens for RT-semi-quantitative PCR, the concentration of extracted RNA was unknown, serving as a limitation. Future studies should therefore measure the concentration of RNA and use the same quantity of RNA for RT-semi quantitative PCR analysis. Additionally, RT-semi quantitative PCR was utilized to detect gene transcription levels; however, this method is not sensitive and requires more RNA input compared with quantitative PCR, which is widely used to detect gene transcription.
The purpose of the present study was to qualitatively detect gene expression. In order to ensure the accuracy of the test results, the skin sample must be large enough (more than $7 \mathrm{mg}$ for each patient) for the RNA to be evaluated.

\section{Acknowledgements}

Not applicable.

\section{Funding}

The current study was supported by the Health and Family Planning Commission of Wuhan municipality (grant no. WX16D08).

\section{Availability of data and materials}

The datasets created during and/or analysed during the current study available from the corresponding author on reasonable request.

\section{Authors' contributions}

WX contributed to conception and design, acquistition of data, and was a major contribution in drafting the manuscript. XW acquired and analyzed the patient data, and detected the tissue RNA. Both authors read and approve the final manuscript.

\section{Ethics approval and consent to participate}

The present study was approved by the Medical Ethics Committee of Wuhan Third Hospital \& Tongren Hospital of Wuhan University (Wuhan, China). Written informed consent was obtained from all participants.

\section{Patient consent for publication}

All patients and healthy volunteers consented to the publication of their images.

\section{Competing interests}

The authors declare that they have no competing interests.

\section{References}

1. Manga P, Elbuluk N and Orlow SJ: Recent advances in understanding vitiligo. F1000Res 5: F1000, 2016.

2. Bacigalupi RM, Postolova A and Davis RS: Evidence-based, non-surgical treatments for vitiligo: A review. Am J Clin Dermatol 13: 217-237, 2012.

3. Lai YC, Yew YW, Kennedy C and Schwartz RA: Vitiligo and depression: A systematic review and meta-analysis of observational studies. Br J Dermatol 177: 708-718, 2017.

4. Boniface K, Seneschal J, Taïeb A and Merched A: Vitiligo therapy: Restoring immune privilege? Exp Dermatol 26: 635-636, 2017.

5. Patel NS, Paghdal KV and Cohen GF: Advanced treatment modalities for vitiligo. Dermatol Surg 38: 381-391, 2012.

6. Ghafourian A, Ghafourian S, Sadeghifard N, Mohebi R, Shokoohini Y, Nezamoleslami S and Hamat RA: Vitiligo: Symptoms, pathogenesis and treatment. Int J Immunopathol Pharmacol 27: 485-489, 2014. 
7. Tsatmali M, Ancans $\mathrm{J}$ and Thody AJ: Melanocyte function and its control by melanocortin peptides. J Histochem Cytochem 50: $125-133,2002$

8. Eskandani M, Golchai J, Pirooznia N and Hasannia S: Oxidative stress level and tyrosinase activity in vitiligo patients. Indian J Dermatol 55: 15-19, 2010.

9. Zhu PY, Yin WH, Wang MR, Dang YY and Ye XY: Andrographolide suppresses melanin synthesis through Akt/GSK3 $/ / \beta$-catenin signal pathway. J Dermatol Sci 79: 74-83, 2015.

10. Nishimura EK, Jordan SA, Oshima H, Yoshida H, Osawa M, Moriyama M, Jackson IJ, Barrandon Y, Miyachi Y and Nishikawa S: Dominant role of the niche in melanocyte stem-cell fate determination. Nature 416: 854-860, 2002.

11. Rodrigues M, Ezzedine K, Hamzavi I, Pandya AG, Harris JE and Vitiligo Working Group: Current and emerging treatments for vitiligo. J Am Acad Dermatol 77: 17-29, 2017.

12. Parsad D, Pandhi R, Dogra S and Kumar B: Clinical study of repigmentation patterns with different treatment modalities and their correlation with speed and stability of repigmentation in 352 vitiliginous patches. J Am Acad Dermatol 50: 63-67, 2004

13. Arrunátegui A, Arroyo C, Garcia L, Covelli C, Escobar C, Carrascal E and Falabella R: Melanocyte reservoir in vitiligo. Int J Dermatol 33: 484-487, 1994.

14. Liu XM,Zhou Q, Xu SZ, Wakamatsu K and Lei TC: Maintenance of immune hyporesponsiveness to melanosomal proteins by DHICA-mediated antioxidation: Possible implications for autoimmune vitiligo. Free Radic Biol Med 50: 1177-1185, 2011.

15. Jiang S, Liu XM, Dai X, Zhou Q, Lei TC, Beermann F, Wakamatsu K and Xu SZ: Regulation of DHICA-mediated antioxidation by dopachrome tautomerase: Implication for skin photoprotection against UVA radiation. Free Radic Biol Med 48: $1144-1151,2010$

16. Budania A, Parsad D, Kanwar AJ and Dogra S: Comparison between autologous noncultured epidermal cell suspension and suction blister epidermal grafting in stable vitiligo: A randomized study. Br J Dermatol 167: 1295-1301, 2012.
17. Maleki M, Banihashemi M and Sanjari V: Efficacy of suction blister epidermal graft without phototherapy for locally stable and resistant vitiligo. Indian J Dermatol 57: 282-284, 2012.

18. Botchkareva NV, Botchkarev VA and Gilchrest BA: Fate of melanocytes during development of the hair follicle pigmentary unit. J Investig Dermatol Symp Proc 8: 76-79, 2003.

19. Benzekri L, Ezzedine K and Gauthier Y: Vitiligo potential repigmentation index: A simple clinical score that might predict the ability of vitiligo lesions to repigment under therapy. Br J Dermatol 168: 1143-1146, 2013

20. Goldstein NB, Koster MI, Hoaglin LG, Spoelstra NS, Kechris KJ Robinson SE, Robinson WA, Roop DR, Norris DA and Birlea SA: Narrow band ultraviolet B treatment for human vitiligo is associated with proliferation, migration, and differentiation of melanocyte precursors. J Invest Dermatol 135: 2068-2076, 2015.

21. Mou Y, Jiang X, Du Y and Xue L: Intelligent bioengineering in vitiligo treatment: Transdermal protein transduction of melanocyte-lineage-specific genes. Med Hypotheses 79: 786-789, 2012.

22. Kawakami A and Fisher DE: The master role of microphthalmia-associated transcription factor in melanocyte and melanoma biology. Lab Invest 97: 649-656, 2017.

23. Guo J, Zhang JF, Wang WM, Cheung FW, Lu YF, Ng CF, Kung HF and Liu WK: MicroRNA-218 inhibits melanogenesis by directly suppressing microphthalmia-associated transcription factor expression. RNA Biol 11: 732-741, 2014

24. Cichorek M, Wachulska M, Stasiewicz A and Tymińska A Skin melanocytes: Biology and development. Postepy Dermatol Alergol 30: 30-41, 2013

This work is licensed under a Creative Commons Attribution-NonCommercial-NoDerivatives 4.0 International (CC BY-NC-ND 4.0) License. 